\title{
Fork-tip needle biopsy versus fine-needle aspiration in endoscopic ultrasound-guided sampling of solid pancreatic masses: a randomized crossover study
}

\section{(ㄷ)(1) $(2) \Theta$}

\author{
Authors \\ Mark Egan ${ }^{5}$, Paul Bassett ${ }^{6}$, Beate Haugk ${ }^{5}$ \\ Institutions \\ 1 HPB Unit, Freeman Hospital, Newcastle upon Tyne \\ Hospitals NHS Foundation Trust, Newcastle upon Tyne, \\ United Kingdom \\ 2 Translational and Clinical Research Institute, Newcastle \\ University, Newcastle upon Tyne, United Kingdom \\ 3 Department of Gastroenterology, John Radcliffe \\ Hospital, Oxford University Hospitals NHS Trust, Oxford, \\ United Kingdom \\ 4 Population Health Sciences Institute, Newcastle \\ University, Newcastle upon Tyne, United Kingdom \\ 5 Department of Cellular Pathology, Royal Victoria \\ Infirmary, Newcastle upon Tyne Hospitals NHS \\ Foundation Trust, Newcastle upon Tyne, United \\ Kingdom \\ 6 Statsconsultancy, Amersham, United Kingdom
}

Kofi W. Oppong ${ }^{1,2}$, Noor L. H. Bekkali ${ }^{3}$, John S. Leeds ${ }^{1,4}$, Sarah J. Johnson ${ }^{5}$, Manu K. Nayar ${ }^{1}$, Antony Darné5,

submitted 16.8.2019

accepted after revision 21.1 .2020

\section{Bibliography}

DOI https://doi.org/10.1055/a-1114-5903

Published online: 11.3.2020 | Endoscopy 2020; 52: 454-461

(c) Georg Thieme Verlag KG Stuttgart · New York

ISSN 0013-726X

Corresponding author

Kofi W. Oppong, BM, FRCP, HPB Unit Freeman Hospital, Newcastle upon Tyne NE7 7DN, United Kingdom

Fax: +44-191-2231249

Kofi.oppong@nhs.net

\# Supplementary material

Online content viewable at:

https://doi.org/10.1055/a-1114-5903

\section{ABSTRACT}

Background A novel fork-tip fine-needle biopsy (FNB) needle has recently been introduced for endoscopic ultrasound (EUS)-guided sampling. The aim of this study was to compare the performance of fork-tip FNB histology and standard fine-needle aspiration (FNA) cytology in the diagnosis of solid pancreatic masses.

Methods A randomized crossover study was performed in patients referred for EUS-guided sampling. Three passes were taken with each needle in a randomized order. Only samples reported as diagnostic of malignancy were considered positive. The primary end point was the sensitivity of diagnosis of malignancy. Secondary end points included the amount of sample obtained, ease of diagnosis, duration of tissue sampling, pathologist viewing time, and cost.

Results 108 patients were recruited. Median age was 69 years (range $30-87$ ) and 57 were male; $85.2 \%$ had a final diagnosis of malignancy. There were statistically significant differences in sensitivity ( $82 \%$ [ $95 \%$ confidence interval (Cl) $72 \%$ to $89 \%$ ] vs. $71 \%$ [ $95 \% \mathrm{Cl} 60 \%$ to $80 \%$ ]), accuracy $(84 \%$ [ $95 \% \mathrm{Cl} 76 \%$ to $91 \%$ ] vs. $75 \%$ [ $95 \% \mathrm{Cl} 66 \%$ to $83 \%$ ]), proportion graded as a straightforward diagnosis (69\% [95\%Cl 60 $\%$ to $78 \%$ ] vs. $51 \%$ [ $95 \% \mathrm{Cl} 41 \%$ to $61 \%]$ ), and median pathology viewing time (188 vs. 332 seconds) $(P<0.001)$ between FNB and FNA needles, respectively. There was no significant difference in cost between an FNB or FNA strategy. Conclusion The diagnostic performance of the fork-tip FNB needle was significantly better than that of FNA; it was associated with ease of diagnosis, shorter pathological viewing times, and was cost neutral.

\section{Introduction}

Tissue acquisition by means of endoscopic ultrasound (EUS)guided fine-needle aspiration (FNA) is the standard of care for diagnosing pancreatic malignancy. Systematic reviews and meta-analyses [1,2] have reported pooled sensitivity of $86.8 \%$ and $85 \%$, respectively. However, reported sensitivity varies and some studies that have used strict cytological criteria and accounted for inadequate samples have documented less im- 
pressive diagnostic performance [3-5]. In an international survey, only one third of endosonographers reported a sensitivity for the diagnosis of solid mass lesions of $>80 \%$ [6]. Needle size, aspiration technique (suction/no-suction, slow stylet pull), and the use of rapid onsite evaluation (ROSE) [7] have all been investigated as means of improving diagnostic performance, without consistent benefit. EUS-guided fine-needle biopsy (FNB) with acquisition of tissue cores for histological assessment offers the theoretical benefit of preserved tissue architecture, improved sample adequacy, and therefore better diagnostic performance. However, the majority of studies with the firstgeneration reverse bevel needle (ProCore; Cook Medical, Bloomington, Indiana, USA) have found no diagnostic benefit $[8,9]$.

Recently, a new core biopsy needle with a novel fork tip (SharkCore FNB needle; Medtronic, Watford, UK) has been introduced. Initial case series have suggested improved diagnostic performance compared with FNA $[10,11]$ and first-generation core biopsy [12] needles. Prospective randomized studies to investigate this new fork-tip needle vs. FNA needles are lacking. Therefore, the aim of our study was to address this question in a randomized crossover study.

\section{Methods}

\section{Study design and patient population}

The study recruited consecutive patients attending for EUSguided tissue sampling of solid pancreatic masses in a tertiary pancreatic cancer center between May 2017 and June 2018. Written informed consent was obtained from all patients for the procedure. The study was carried out in accordance with the Declaration of Helsinki and was approved by the North East-Newcastle and North Tyneside 1 Research Ethics Committee (reference 17/NE/001). The study is registered at ClinicalTrials.gov (NCT03532347), and is reported according to the Standards for Reporting Diagnostic Accuracy Studies (STARD) and the Consolidated Standards of Reporting Trials (CONSORT) statements (see the online-only Supplementary material).

Adult patients ( $\geq 18$ years) with a suspicion of pancreatic mass based on prior cross-sectional imaging were eligible. Exclusion criteria included cystic lesions without a significant solid component, and any contraindication to pancreatic biopsy. The presence of a self-expandable metal stent in patients with a mass in the head of the pancreas was an initial exclusion criterion, but this was removed by protocol amendment part way through the study.

\section{EUS-guided sampling}

All procedures were performed under conscious sedation using combinations of intravenous midazolam and pethidine, with standard cardiorespiratory monitoring. Pentax echoendoscopes (EG3270UK and EG3870UTX; Pentax, Slough, UK) and Hitachi ultrasound workstations (Preirus; Hitachi Medical Systems, Wellingborough, UK) were used. A single Beacon EUS delivery device (Medtronic Ltd., Watford, UK) was used with 25gauge and 22-gauge needles for transduodenal and transgastric biopsy, respectively. For all procedures, $10 \mathrm{~mL}$ suction and fanning were used. The needle was moved backward and for- ward within the lesion for a minimum of 10 throws. Suction was stopped prior to withdrawal of the needle.

Needles were used in a randomized order. Patients were randomly allocated to an initial three passes with the FNA needle (Beacon FNA needle; Medtronic) or FNB needle (SharkCore FNB needle; Medtronic) and then crossed over to the other needle. Randomization was via a computer-generated list of allocations produced in advance of the study and referred to after participant recruitment. After the initial three passes, the first needle was discarded and the other needle was affixed to the delivery device, which remained attached to the echoendoscope, and a further three passes were made. Procedures were timed using a stopwatch.

Samples were expelled from the needles in a standardized manner. The stylet was first introduced and then the needle was flushed with a few milliliters of normal saline. For FNA, a drop from the needle was expelled onto a glass slide and a single spread slide was made from each pass. The remaining aspirate was expelled into a container with approximately $5 \mathrm{~mL}$ of BD CytoRich Red Preservative solution (BD; Bioscience Healthcare, Nottingham, UK). The air-dried slides, including the draw slide, were fixed in Reastain Quick Diff fix solution (Gamidor Technical Services, Southmead Park UK) to be stained using the Reastain Quick Diff stains. In the laboratory, any clots or microbiopsies present in the CytoRich solution were removed and placed in formalin and subsequently embedded in paraffin. A single $4-\mu \mathrm{m}$ section was cut and stained with hematoxylin and eosin (H\&E).

For all specimens, the remaining CytoRich specimen was processed for staining by SurePath Papanicolaou staining method (Bioscience Healthcare).

All samples from the FNB needle were placed in a single container of $10 \%$ neutral buffered formalin. Samples were subsequently embedded in paraffin. A single 4- $\mu \mathrm{m}$ section was cut and stained with $\mathrm{H} \& \mathrm{E}$.

Additional stains were performed at the discretion of the pathologist if further information for diagnosis was required.

\section{Follow-up}

All procedures were performed as day cases. Patients were observed in the endoscopy recovery area as per routine recovery protocol until fully awake and allowed home if asymptomatic. Patients were contacted by a research nurse 7 days after the procedure to ascertain the occurrence of any post-procedure adverse events. Serious adverse events were defined as complications resulting in a physician visit or an episode of hospitalization or extension of an existing hospital stay, significant disability or death [13].

\section{Pathology}

The FNA samples were reported by one of two specialist cytopathologists and the FNB by one of two pancreatic histopathologists. The pathologists were blinded to the report of the other sampling method. All samples were reported with a prose report describing the findings, with an interpretation and final diagnostic category: inadequate, benign, atypical, suspicious of malignancy or malignant. The pathologist timed microscope 
interpretation using a stopwatch (including for any subsequent immunostaining); only the time spent examining slides was measured. A novel, simple three-tier score to allow comparison of cytology and histology samples with regard to amount of diagnostic material and ease of diagnosis was used (see Supplementary material).

\section{Definitions and end points}

The primary end point was diagnostic performance of the forktip FNB needle compared with the standard FNA needle. The primary end point was assessed using "strict" criteria as follows; samples reported as benign, atypical, and suspicious were categorized as negative for malignancy and only samples reported as malignant were categorized as positive for malignancy. For the purposes of the study, low grade malignant tumors (i.e. gastrointestinal stromal tumors, lymphoma, and neuroendocrine tumors) were considered malignant. Specimens that contained inadequate material were not excluded from primary analysis and were considered as negative for malignancy. A secondary "less strict" analysis was also performed excluding inadequate samples and categorizing suspicious samples as diagnostic of malignancy.

A conventional "malignancy" analysis was performed purely for the differentiation of malignancy from benign (i.e. correct classification as benign or malignant was the only requirement). A "specific diagnosis" analysis was also performed; for this, a specific tissue diagnosis that matched the final diagnosis was required. Diagnostic performance was expressed using standard performance parameters including sensitivity, specificity, and overall accuracy. Secondary end points were duration of sampling time (in seconds), duration of pathologist viewing time (in seconds), amount of diagnostic material, ease of diagnosis, and cost analysis.

The final gold standard diagnosis for malignancy required either unequivocal malignant pathology obtained by EUS sampling, surgical resection or alternative biopsy. For nonoperated patients with nondiagnostic tissue sampling, clinical and radiological disease progression consistent with malignancy at 6month follow-up was required.

Benign classification required nonmalignant and nonsuspicious tissue sampling and follow-up of at least 6 months with a consistent clinical picture and no evidence of malignancy on interval imaging.

\section{Statistical analysis}

Sample size

The sample size was calculated in order to show a difference in sensitivity for the diagnosis of malignancy of $14 \%$ between groups based on prior data from our unit ( $92 \% \mathrm{FNB}, 78 \% \mathrm{FNA}$ ). The percentage of patients having discordant results for the two needles was estimated to be $22 \%$. Using a $5 \%$ significance level and $80 \%$ power, we calculated that 86 malignant cases were required and estimated that malignant cases would account for $85 \%$ of all recruited cases. It was also assumed that there would be an attrition rate of $5 \%$ due to loss of patients during follow-up. After inflating the sample size to account for both factors, we calculated that 107 patients were required. To allow for a balanced design the target sample size was set at 108.

\section{Data analyses}

Baseline characteristics of the patient population, pancreatic mass lesions, technical details, and procedure outcomes were summarized as means with standard deviation (SD) or medians with interquartile range (IQR) for continuous data, and as frequencies and proportions for categorical data. The data analysis allowed for the fact that each patient had two results, and that two outcomes from the same patient are likely to be more similar than two results from different patients. The analysis also factored in that outcomes in the first period (i.e. first needle used) may be different from those in the second period. All analyses were performed using multilevel logistic regression or multilevel linear regression (STATA version 15.1; StataCorp, College Station, Texas, USA). Two-level models were used, with individual results nested within patients. Fixed terms were included in the model for the period (first or second needle) and the needle used (FNA or FNB), while patient was used as a random factor.

An initial analysis examined whether the differences between needles varied depending on whether they were from the first or second period (needle by period interaction) as this could indicate a carryover effect from the first to second period. If this interaction was not statistically significant, the interaction term was omitted, and the differences between needles were assessed using data from both periods.

Analysis of diagnostic performance, amount of material, and ease of diagnosis was performed. For amount of material and ease of diagnosis, two different outcomes were considered: first, the proportion scored as 3 , and second the proportion scored as 2 or 3 . Tissue sampling duration and pathology viewing time were compared. All tests were two-sided and $P$ values $<0.05$ were considered statistically significant.

\section{Cost analysis}

The cost of an FNB sampling strategy was compared with an FNA sampling strategy. The cost was based on the England National Health Service (NHS) 2017/18 tariff for an EUS tissue sampling procedure (€639). This tariff includes the cost of any of the accessories used, pathological processing, and reporting. The standard NHS cost was $€ 187$ for the FNA needle and $€ 263$ for the fork-tip needle. To better reflect the cost of needle choice to the health economy for the purposes of the study, the "cost" of the procedure was assumed to be the NHS tariff plus the cost of needle.

If a nondiagnostic result was obtained, it was assumed that a single repeat procedure would be performed for that patient using the same type of needle. Bootstrap methods were used to obtain the confidence interval $(\mathrm{Cl})$ for the cost difference between needles; 100 bootstrap samples were used in the calculations. 


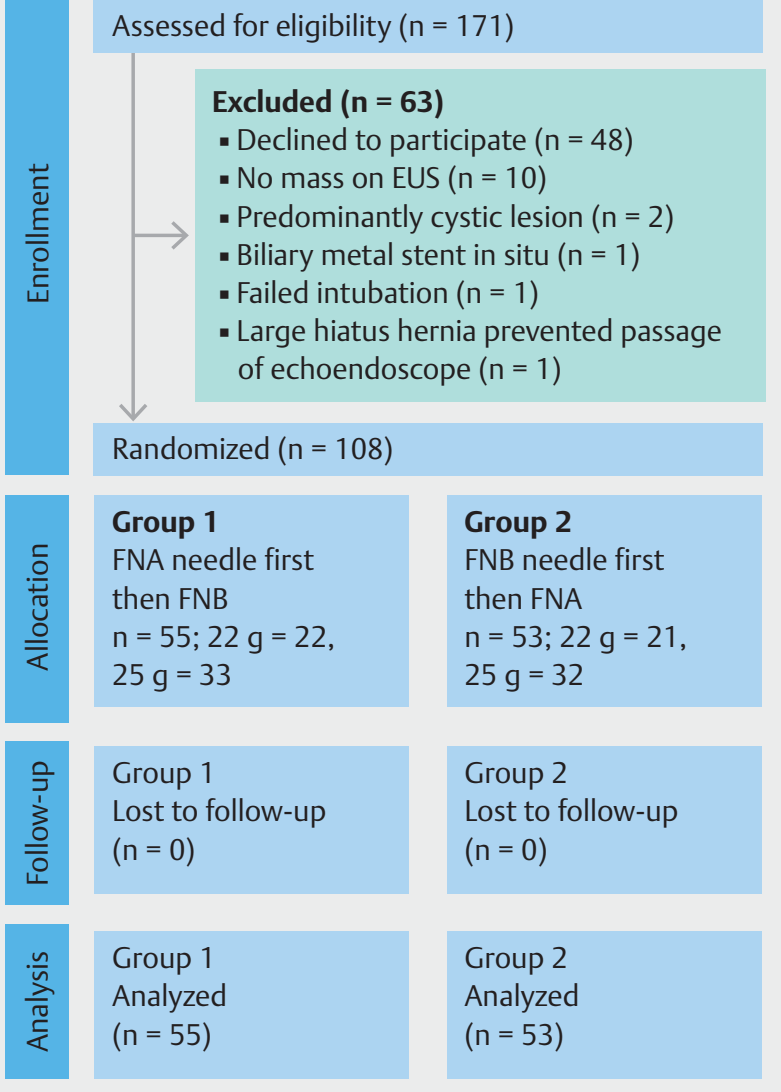

- Fig. 1 CONSORT study flow diagram. Enrollment and outcomes. CONSORT, Consolidated Standards of Reporting Trials; FNA, fine-needle aspiration; FNB, fine-needle biopsy.

\section{Results}

A total of 171 patients were assessed for eligibility, 63 were excluded, and 108 (57 men, 51 women) with a median age of 69 years (range $30-87$ ) were randomized ( Fig. 1 ). Five patients had a self-expandable metal stent in situ. Lesion characteristics and final diagnoses are shown in $>$ Table 1. Table 2 shows the pathological diagnostic category. There was no difference between the FNB and FNA needles in the proportion of malignant cases reported as unsatisfactory (3.3\% vs $4.3 \%$, respectively); however, there was a difference in the proportion of malignant cases categorized as suspicious (6.5\% vs. $18.5 \%$, respectively).

\section{Diagnostic performance}

The results ( $\triangleright$ Table 3 ) suggested that for all diagnostic outcomes, there was no significant needle by period interaction. Therefore, the needle effect was quantified using the data from both periods combined.

There was a significant difference in diagnostic performance between the needles. Sensitivity for FNB was $82 \%(95 \% \mathrm{Cl} 72 \%$ to $89 \%$ ) compared with $71 \%(95 \% \mathrm{Cl} 60 \%$ to $80 \%)$ for FNA; the odds of detecting a malignancy with an FNB needle were $>3$ times higher than the odds for an FNA needle (odds ratio [OR] $3.23,95 \% \mathrm{Cl} 1.12$ to 9.38 ).

- Table 1 Lesion characteristics and final diagnosis.

\begin{tabular}{|c|c|}
\hline Cases, $\mathbf{n}$ & 108 \\
\hline Size of mass, median (IQR), mm & $25(19-34.5)$ \\
\hline \multicolumn{2}{|l|}{ Location, n (\%) } \\
\hline - Head/uncinate & $62(57.4)$ \\
\hline - Neck & $12(11.1)$ \\
\hline - Body & $21(19.4)$ \\
\hline - Tail & $13(12.0)$ \\
\hline \multicolumn{2}{|l|}{ Diagnosis, n (\%) } \\
\hline Malignant & $92(85.2)$ \\
\hline - Pancreatic ductal adenocarcinoma & $69(63.9)$ \\
\hline - Neuroendocrine & $14(12.9)$ \\
\hline - Metastatic renal cell carcinoma & $4(3.7)$ \\
\hline - Lymphoma & $2(1.9)$ \\
\hline - Cholangiocarcinoma & $1(0.92)$ \\
\hline - Solid pseudopapillary neoplasm & $1(0.92)$ \\
\hline - Metastatic breast adenocarcinoma & $1(0.92)$ \\
\hline Benign & $16(14.8)$ \\
\hline - Pancreatitis & $9(8.3)$ \\
\hline - Heterotopic spleen & $2(1.9)$ \\
\hline - Autoimmune pancreatitis & $2(1.9)$ \\
\hline - Benign peripheral nerve tumour & $1(0.92)$ \\
\hline - Unspecified & $2(1.9)$ \\
\hline
\end{tabular}

The needles were found to vary significantly in terms of accuracy for specific diagnosis. Accuracy was 79\% (95\% Cl $70 \%$ to $86 \%$ ) for FNB compared with $64 \%$ ( $95 \% \mathrm{Cl} 54 \%$ to $73 \%$ ) for FNA. The odds of an accurate specific diagnosis were $>4$ times higher with the FNB needle compared with the FNA needle (OR 4.79, $95 \% \mathrm{Cl} 1.67$ to 13.7$)$.

\section{Analysis using less strict criteria}

There was no significant difference in diagnostic performance for malignancy or specific diagnosis when the "less strict" criteria were used (Table $1 \mathrm{~s}$ ).

\section{Duration of sampling and pathology viewing}

- Table 4 summarizes the results. The sampling time was found to be significantly shorter for the FNB needle compared with the FNA needle: mean 710 (SD 171) seconds vs. 759 (SD 144) seconds $(P=0.001)$. Pathology viewing times were also significantly shorter for FNB compared with FNA needles: median 188 seconds (IQR $56-330)$ vs. 332 seconds (IQR 212-411) $(P$ $<0.001)$. 
- Table 2 Pathological classification of samples.

\begin{tabular}{|c|c|c|c|c|c|}
\hline Final diagnosis & Unsatisfactory, $\mathrm{n}(\%)$ & Benign, n (\%) & Atypical, n (\%) & Suspicious, n (\%) & Malignant, n (\%) \\
\hline \multicolumn{6}{|l|}{ Malignant ( $n=92$ ) } \\
\hline - FNB & $3(3.3)$ & $2(2.2)$ & $6(6.5)$ & $6(6.5)$ & $75(81.5)$ \\
\hline - FNA & $4(4.3)$ & 0 & $6(6.5)$ & $17(18.5)$ & $65(70.7)$ \\
\hline \multicolumn{6}{|l|}{ Benign $(n=16)$} \\
\hline - FNB & $6(37.5)$ & $9(56.2)$ & $1(6.2)$ & 0 & 0 \\
\hline - FNA & $5(31.2)$ & $7(43.7)$ & $4(25.0)$ & 0 & 0 \\
\hline
\end{tabular}

- Table3 Diagnostic performance.

\begin{tabular}{|c|c|c|c|c|c|c|c|}
\hline \multirow[t]{2}{*}{ Outcome } & \multirow{2}{*}{$\mathrm{FN} / \mathrm{N}$} & \multirow[b]{2}{*}{$\%(95 \% \mathrm{Cl})$} & \multicolumn{2}{|l|}{ FNA } & \multirow{2}{*}{$\begin{array}{l}\text { NxP } \\
\text { Interaction } \\
\text { P value }\end{array}$} & \multicolumn{2}{|c|}{ Effect of needle type } \\
\hline & & & $n / N$ & $\%(95 \% \mathrm{Cl})$ & & OR $(95 \% \mathrm{Cl})^{2}$ & $P$ value \\
\hline \multicolumn{8}{|l|}{ Diagnosis of malignancy } \\
\hline - Accuracy & $91 / 108$ & 84 (76 to 91 ) & $81 / 108$ & 75 (66 to 83 ) & 0.26 & $\begin{array}{l}3.41 \\
(1.12 \text { to } 10.4)\end{array}$ & 0.03 \\
\hline - Sensitivity & $75 / 92$ & 82 (72 to 89$)$ & $65 / 92$ & 71 (60 to 80$)$ & 0.25 & $\begin{array}{l}3.23 \\
(1.12 \text { to } 9.38)\end{array}$ & 0.03 \\
\hline - Specificity & $16 / 16$ & 100 (79 to 100$)$ & $16 / 16$ & 100 (79 to 100$)$ & - & - & - \\
\hline $\begin{array}{l}\text { Positive predictive } \\
\text { value }\end{array}$ & $75 / 75$ & 100 (94 to 100$)$ & $65 / 65$ & 100 (94 to 100$)$ & - & - & - \\
\hline $\begin{array}{l}\text { - Negative predictive } \\
\text { value }\end{array}$ & $16 / 33$ & 48 (31 to 66$)$ & $16 / 43$ & 37 (22 to 53$)$ & 0.78 & $\begin{array}{l}1.62 \\
(0.64 \text { to } 4.10)\end{array}$ & 0.31 \\
\hline \multicolumn{8}{|l|}{ Specific diagnosis } \\
\hline - Accuracy & $85 / 108$ & 79 (70 to 86$)$ & $69 / 108$ & 64 (54 to 73$)$ & 0.13 & $\begin{array}{l}4.79 \\
(1.67 \text { to } 13.7)\end{array}$ & 0.004 \\
\hline - Accuracy ${ }^{3}$ & $10 / 16$ & 63 (35 to 85$)$ & $4 / 16$ & 25 (7 to 52$)$ & - & - & - \\
\hline \multicolumn{8}{|c|}{$\begin{array}{l}\text { FNB, fine-needle biopsy; FNA, fine-needle aspiration; NxP, needle by period; } \mathrm{Cl} \text {, confidence interval; OR, odds ratio. } \\
{ }^{1} \text { Analysis to examine whether the differences between needles varied depending on whether they were used first or second. } \\
{ }^{2} \text { OR calculated as odds for FNB relative to odds for FNA. } \\
{ }^{3} \text { Analysis based on patients with a benign final diagnosis only. }\end{array}$} \\
\hline
\end{tabular}

\section{Amount of material, ease of diagnosis, and cost}

The results for amount of material and ease of diagnosis are summarized in > Table 5. A significantly higher proportion of FNB cases were categorized as 3 (abundant for diagnosis): $58 \%$ ( $95 \% \mathrm{Cl} 48 \%$ to $68 \%$ ) vs. $44 \%$ (95\%Cl $35 \%$ to $54 \%$ ) for FNA; however, this was associated with a significant needle by period interaction. There was no difference when only data from the first period were analyzed. For ease of diagnosis, a significantly higher proportion of FNB cases were graded as 3 (straightforward diagnosis): $69 \%$ ( $95 \% \mathrm{Cl} 60 \%$ to $78 \%$ ) vs. $51 \%(95 \% \mathrm{Cl} 41$ $\%$ to $61 \%$ ) for FNA. There was no significant difference in cost: mean $€ 1044$ (SD 330) vs. €1033 (SD359) for FNB vs. FNA, respectively. The mean difference was $12(95 \% \mathrm{Cl}-55$ to $95 ; P$ $=0.74)$.

\section{Adverse events}

Serious adverse events occurred in four patients within 7 days (3.7\%): two developed cholangitis (one of whom underwent endoscopic retrograde cholangiopancreatography immediately following EUS tissue sampling, and the other had a pre-existing plastic biliary stent), one patient developed pancreatitis, and one patient was hospitalized for 24 hours with severe abdominal pain.

\section{Discussion}

In this prospective, randomized, crossover study comparing the fork-tip FNB and FNA needles in the sampling of solid pancreatic masses, the FNB needle was more sensitive than the FNA nee- 
- Table4 Sampling time and pathology viewing time.

\begin{tabular}{|c|c|c|c|c|c|c|c|}
\hline \multirow[t]{2}{*}{ Outcome } & \multirow{2}{*}{\multicolumn{2}{|c|}{ FNB }} & \multirow{2}{*}{\multicolumn{2}{|c|}{ FNA }} & \multirow{2}{*}{$\begin{array}{l}\mathrm{NxP} \\
\text { interaction }{ }^{1} \\
P \text { value }\end{array}$} & \multicolumn{2}{|c|}{ Effect of needle type } \\
\hline & & & & & & \multirow{2}{*}{$\begin{array}{l}\text { Mean/Ratio } \\
(95 \% \mathrm{Cl}) \\
\text { Mean }-49 \\
(-79 \text { to }-19)^{3}\end{array}$} & \multirow{2}{*}{$\begin{array}{l}P \text { value } \\
0.001\end{array}$} \\
\hline Sampling time, seconds & $n=108$ & $\begin{array}{l}\text { Mean } 710 \\
\text { (SD 171) }\end{array}$ & $\mathrm{n}=106^{2}$ & $\begin{array}{l}\text { Mean } 759 \\
(\text { SD 144) }\end{array}$ & 0.94 & & \\
\hline $\begin{array}{l}\text { Pathology viewing time, } \\
\text { seconds }\end{array}$ & $\mathrm{n}=90^{4}$ & $\begin{array}{l}\text { Median } 188 \\
\text { (IQR 56-330) }\end{array}$ & $n=89^{5}$ & $\begin{array}{l}\text { Median } 332 \\
\text { (IQR 212- } \\
411)\end{array}$ & 0.30 & $\begin{array}{l}\text { Ratio } 0.49 \\
(0.41 \text { to } 0.60)^{6}\end{array}$ & $<0.001$ \\
\hline \multicolumn{8}{|c|}{$\begin{array}{l}\text { FNB, fine-needle biopsy; FNA, fine-needle aspiration; NxP, needle by period; Cl, confidence interval; IQR, interquartile range. } \\
{ }^{1} \text { Analysis to examine whether the differences between needles varied depending on whether they were used first or second. } \\
22 \text { patients omitted from analysis as data unavailable. } \\
{ }^{3} \text { Mean difference calculated as values for FNB minus values for FNA. } \\
{ }^{4} 18 \text { patients omitted from analysis. } \\
519 \text { patients omitted from analysis. } \\
{ }^{6} \text { Ratio calculated as values for FNB divided by values for FNA. }\end{array}$} \\
\hline
\end{tabular}

dle for the diagnosis of pancreatic malignancy ( $82 \%$ vs. $71 \%$ ) when using strict criteria. Additionally, FNB had significantly shorter sampling and pathology viewing times, as well as greater ease of diagnosis reported by the pathologists. Under "less strict” criteria, with suspicious samples categorized as diagnostic of malignancy, the difference in diagnostic performance was eliminated. However, a suspicious diagnosis is not acceptable by most oncologists for initiation of chemotherapy and such patients would not be eligible for recruitment into chemotherapy trials.

EUS core biopsy needles have been designed to provide larger tissue samples with preserved tissue architecture that are adequate for histological assessment. If achieved, this would enable speedier, more accurate diagnosis, in addition to the potential to conduct the more detailed histological subtyping and genetic analysis of tumors required for personalized therapies. Histological assessment should also facilitate the definitive diagnosis of benign conditions such as autoimmune pancreatitis and chronic pancreatitis, which are challenging to make with FNA.

The reverse bevel needle was the first widely used core biopsy needle; however, this has not shown unequivocal superiority to FNA. A recent large prospective study [14] reported significantly higher diagnostic yield with the reverse bevel needle compared with FNA for pancreatic lesions; however, it is unclear whether this equated to better diagnostic accuracy or rather more frequent diagnostic histological samples. In an earlier study by Vanbiervliet et al., in addition to finding no difference in diagnostic performance, the authors reported better overall sample quality with the FNA needle [15]. Two meta-analyses $[8,16]$ reported no significant difference in sensitivity or acquisition of cores, while another [17] reported the core needles to have better specimen adequacy and diagnostic accuracy.

The SharkCore needle has a fork tip, opposing bevel design with six cutting surfaces designed to acquire architecturally intact tissue and minimize tissue disruption. Initial retrospective studies documented high pathological yield with fewer passes $[10,11]$ and the needle significantly outperformed the reverse bevel with an adjusted OR of 7.93 [12]. A further retrospective study found no difference in diagnostic performance between the fork-tip needle and FNA needle, although the fork-tip needle yielded a significantly higher proportion of tissue that was adequate for histological evaluation and ancillary diagnostic techniques [18]. A recent multicenter prospective study using the 25-gauge fork-tip needle for sampling solid pancreatic masses reported the presence of cores in $67 \%$ of samples [19]. In a large retrospective study of 3020 patients, $71.3 \%$ of whom were undergoing sampling of a pancreatic mass using FNA or second-generation core biopsy needles (either Franseen-Acquire, Boston Scientific Corp - or fork-tip), the median number of passes required for diagnostic adequacy on ROSE was significantly lower for FNB compared with FNA, and diagnostic yield on cell block was superior with FNB [20]. The superior performance of FNB over FNA was observed for both pancreatic and nonpancreatic lesions. A multicenter retrospective study of 22-gauge fork-tip and 22-gauge FNA needles in patients with solid pancreatic mass lesions found no significant difference in diagnostic performance but a significantly reduced number of passes to obtain diagnostic tissue [21]; ROSE was available for all procedures in this study. Another retrospective study of primarily pancreas and gut wall lesions without on-site cytopathology also reported fewer needle passes with the fork-tip needle [22]; there was, again, no significant difference in sensitivity (fork-tip $89.9 \%$ vs. FNA $81.0 \%$ ). The diagnostic performance of Franseen and fork-tip needles appears to be similar; a recent randomized trial [23] found no significant difference between the two needles in tissue yield, with $>90 \%$ of diagnostic cell blocks.

Conventionally, the diagnostic performance of EUS tissue sampling in pancreatic masses is considered in a binary manner - cancer/noncancer. This reflects the clinical scenario in which the pre-test probability of malignancy is high, and a diagnosis of malignancy will trigger major surgery and/or chemotherapy. However, a significant minority of cases (15\% in the present study) will be benign and a definitive histological diagnosis in this context is of value. First, by enhancing the benign diagnosis beyond one of just "nonmalignant," confidence in the accuracy 
- Table 5 Tissue amount and ease of diagnosis.

\begin{tabular}{|c|c|c|c|c|c|c|c|}
\hline \multirow[t]{2}{*}{ Outcome } & \multicolumn{2}{|l|}{ FNB } & \multicolumn{2}{|l|}{ FNA } & \multirow{2}{*}{$\begin{array}{l}\text { NxP } \\
\text { interaction } \\
\text { P value }\end{array}$} & \multicolumn{2}{|c|}{ Effect of needle type } \\
\hline & $\mathrm{n} / \mathrm{N}$ & $\%(95 \% \mathrm{Cl})$ & $\mathrm{n} / \mathrm{N}$ & $\%(95 \% \mathrm{CI})$ & & OR $(95 \% \mathrm{Cl})^{2}$ & $P$ value \\
\hline \multicolumn{8}{|c|}{ Amount of material classification } \\
\hline - Too scanty for diagnosis - 1 & $11 / 108$ & 10 (5 to 17$)$ & $18 / 108$ & 17 (10 to 25$)$ & & & \\
\hline - Sufficient for diagnosis - 2 & $34 / 108$ & 31 (23 to 41$)$ & $42 / 108$ & 39 (30 to 49$)$ & & & \\
\hline - Abundant for diagnosis - 3 & $63 / 108$ & 58 (48 to 68$)$ & $48 / 108$ & 44 (35 to 54$)$ & & & \\
\hline \multicolumn{8}{|l|}{ Statistical comparison } \\
\hline - Proportion graded 3 & $63 / 108$ & 58 (48 to 68$)$ & $48 / 108$ & $44(35$ to 54$)$ & 0.01 & $\begin{array}{l}2.17 \text { ( } 1.12 \text { to } \\
4.22)\end{array}$ & 0.02 \\
\hline - Proportion graded $3^{3}$ & $27 / 53$ & 51 (37 to 65$)$ & $31 / 55$ & $56(42$ to 70$)$ & - & $\begin{array}{l}0.75 \text { ( } 0.27 \text { to } \\
2.06)\end{array}$ & 0.57 \\
\hline - Proportion graded 2 or 3 & $97 / 108$ & 90 (83 to 95$)$ & $90 / 108$ & 83 (75 to 90$)$ & 0.61 & $\begin{array}{l}2.47 \text { ( } 0.84 \text { to } \\
7.25)\end{array}$ & 0.10 \\
\hline \multicolumn{8}{|l|}{ Ease of diagnosis classification } \\
\hline - Challenging/impossible-1 & $9 / 108$ & $8(4$ to 15$)$ & $12 / 108$ & $11(6$ to 19$)$ & & & \\
\hline - Some difficulty-2 & $24 / 108$ & $22(15$ to 31$)$ & $41 / 108$ & 38 (29 to 48$)$ & & & \\
\hline - Straightforward-3 & $75 / 108$ & 69 (60 to 78$)$ & $55 / 108$ & 51 (41 to 61$)$ & & & \\
\hline \multicolumn{8}{|l|}{ Statistical comparison } \\
\hline - Proportion graded 3 & $75 / 108$ & 69 (60 to 78$)$ & $55 / 108$ & 51 (41 to 61 ) & 0.17 & $\begin{array}{l}3.48 \text { (1.60 to } \\
7.59)\end{array}$ & 0.002 \\
\hline - Proportion graded 2 or 3 & $99 / 108$ & 92 (85 to 96$)$ & $96 / 108$ & 89 (82 to 94$)$ & 0.87 & $\begin{array}{l}1.62 \text { ( } 0.52 \text { to } \\
5.09 \text { ) }\end{array}$ & 0.41 \\
\hline \multicolumn{8}{|c|}{$\begin{array}{l}\text { FNB, fine-needle biopsy; FNA, fine-needle aspiration; NxP, needle by period; Cl, confidence interval; OR, odds ratio. } \\
{ }^{1} \text { Analysis to examine whether the differences between needles varied depending on whether they were used first or second. } \\
2 \text { OR given as odds for FNB relative to odds for FNA. } \\
{ }^{3} \text { Results based on data from first period only. }\end{array}$} \\
\hline
\end{tabular}

of the diagnosis is increased, thereby lessening the likelihood of repeat sampling or inappropriate surgery. Second, a specific histological diagnosis (e.g. autoimmune pancreatitis) will ensure appropriate therapy is initiated promptly.

This study therefore also reported diagnostic performance in terms of requiring a specific tissue diagnosis (malignant or benign) that matched the final diagnosis. Using this threshold, a benign case (e.g. chronic pancreatitis) where the pathology report stated benign/noncancer without establishing a diagnosis of chronic pancreatitis would be classified as inaccurate. Use of this higher threshold resulted in an even greater difference in diagnostic performance between the needles, with an OR of 4.79.

The results reflect the twin benefits of greater amount of tissue and preserved tissue architecture with the FNB needle. Sampling time was also significantly shorter, and reflects the differences in in-room preparation; FNB samples were expelled directly into formaldehyde whereas for each FNA pass, a slide was prepared and the remaining material expelled into a container. However, though statistically significant, this small difference in sampling time is unlikely to be clinically relevant.
The strengths of the present study include its prospective, randomized, crossover design and the blinding of the reporting pathologists to the results with the paired needle. Potential weaknesses include the inability to blind the endosonographers to the needle type; however, adherence to strict protocols in terms of number of passes, throws within the lesion, and use of the fanning technique should have mitigated this bias. The use of separate histopathologists and cytopathologists is another possible weakness as this prevented blinding to the needle type. However, both sets of pathologists had extensive expertise in the reporting of pancreatic histology and cytology, respectively, and due to the very different nature of the pathological processing it would have been impossible for pathologists to be blinded to the nature of the sample they were reporting. The different processing techniques of the samples does raise the possibility that this, and not only the needle, was a factor in the data differences identified; however, any clots or microbiopsies obtained with the FNA needle were processed in the same way as samples from the FNB needle. Additionally, the amount of material and ease of diagnosis were as- 
sessed using a novel nonvalidated scoring system, as existing scoring systems were not suitable for this purpose.

In conclusion, we have demonstrated in a prospective randomized study that the EUS fork-tip biopsy needle yielding a histology specimen delivers substantially better diagnostic performance, better sample amount, ease of reporting, and shorter sampling and pathological viewing times than a standard FNA needle yielding a cytology specimen, and this strategy was cost neutral. This finding provides the first prospective evidence to support the contention that such EUS core biopsy needles should be regarded as the standard of care for tissue acquisition from solid pancreatic masses.

\section{Acknowledgment}

This investigator-initiated study was funded by the Medtronic external research programme. Funding was unrestricted and Medtronic had no influence on the study design, execution, data analysis or manuscript content.

The study was presented as a poster at Digestive Disease Week, San Diego, May 2019, and the British Society of Gastroenterology annual meeting, Glasgow, June 2019.

\section{Competing interests}

Dr. Oppong has received a research grant, travel grant, and speaker fees from Medtronic. Dr. Bekkali has received a travel grant from Boston Scientific. Drs. Nayar and Haugk have received research grants and speaker fees from Medtronic. Drs. Johnson, Leeds, and Darne have received research grants from Medtronic. Dr. Bassett has no conflict of interest.

\section{References}

[1] Puli SR, Bechtold ML, Buxbaum JL et al. How good is endoscopic ultrasound-guided fine-needle aspiration in diagnosing the correct etiology for a solid pancreatic mass? a meta-analysis and systematic review Pancreas 2013; 42: 20-26

[2] Hewitt MJ, McPhail MJW, Possamai L et al. EUS-guided FNA for diagnosis of solid pancreatic neoplasms: a meta-analysis. Gastrointest Endosc 2012; 75: 319-331

[3] Turner BG, Cizginer S, Agarwal D et al. Diagnosis of pancreatic neoplasia with EUS and FNA: a report of accuracy. Gastrointest Endosc 2010; 71: 91-98

[4] van Riet PA, Larghi A, Attili F et al. A multicenter randomized trial comparing a 25-gauge EUS fine-needle aspiration device with a 20gauge EUS fine-needle biopsy device. Gastrointest Endosc 2019; 89: 329-339

[5] Kamata K, Kitano M, Yasukawa S et al. Histologic diagnosis of pancreatic masses using 25-gauge endoscopic ultrasound needles with and without a core trap: a multicenter randomized trial. Endoscopy 2016; 48: 632-638

[6] Dumonceau J-M, Koessler T, van Hooft JE et al. Endoscopic ultrasonography-guided fine needle aspiration: relatively low sensitivity in the endosonographer population. World J Gastroenterol 2012; 18: 23572363
[7] Kandel P, Wallace MB. Advanced EUS guided tissue acquisition methods for pancreatic cancer. Cancers (Basel) 2018; 10: 54

[8] Bang JY, Hawes R, Varadarajulu S. A meta-analysis comparing ProCore and standard fine-needle aspiration needles for endoscopic ultrasound-guided tissue acquisition. Endoscopy 2016; 48: 339-349

[9] Facciorusso A, Bajwa HS, Menon K et al. Comparison between 22G aspiration and 22G biopsy needles for EUS-guided sampling of pancreatic lesions: a meta-analysis. Endosc Ultrasound 2019: doi:10.4103/eus.eus_4_19

[10] Kandel P, Tranesh G, Nassar A et al. EUS-guided fine needle biopsy sampling using a novel fork-tip needle: a case-control study. Gastrointest Endosc 2016; 84: 1034-1039

[11] DiMaio C, Kolb J, Benias P et al. Initial experience with a novel EUSguided core biopsy needle (SharkCore): results of a large North American multicenter study. Endosc Int Open 2016; 4: E974-979

[12] Nayar MK, Paranandi B, Dawwas MF et al. Comparison of the diagnostic performance of 2 core biopsy needles for EUS-guided tissue acquisition from solid pancreatic lesions. Gastrointest Endosc 2017; 85: 1017-1024

[13] Eloubeidi MA, Tamhane A, Varadarajulu S et al. Frequency of major complications after EUS-guided FNA of solid pancreatic masses: a prospective evaluation. Gastrointest Endosc 2006; 63: 622-629

[14] Cheng B, Zhang Y, Chen Q et al. Analysis of fine-needle biopsy vs fineneedle aspiration in diagnosis of pancreatic and abdominal masses: a prospective, multicenter, randomized controlled trial. Clin Gastroenterol Hepatol 2018; 16: 1314-1321

[15] Vanbiervliet G, Napoléon B, Saint Paul M et al. Core needle versus standard needle for endoscopic ultrasound-guided biopsy of solid pancreatic masses: a randomized crossover study. Endoscopy 2014; 46: 1063-1070

[16] Khan MA, Grimm IS, Ali B et al. A meta-analysis of endoscopic ultrasound-fine-needle aspiration compared to endoscopic ultrasoundfine-needle biopsy: diagnostic yield and the value of onsite cytopathological assessment. Endosc Int open 2017; 5: E363-E375

[17] Li H, Li W, Zhou QY et al. Fine needle biopsy is superior to fine needle aspiration in endoscopic ultrasound guided sampling of pancreatic masses. Medicine (Baltimore) 2018; 97: e0207

[18] Jovani M, Abidi WM, Lee LS. Novel fork-tip needles versus standard needles for EUS-guided tissue acquisition from solid masses of the upper GI tract: a matched cohort study. Scand J Gastroenterol 2017; 52: 784-787

[19] Di Leo M, Crinò SF, Bernardoni L et al. EUS-guided core biopsies of pancreatic solid masses using a new fork-tip needle: a multicenter prospective study. Dig Liver Dis 2019; 51: 1275-1280

[20] Bang JY, Kirtane S, Krall K et al. In memoriam: Fine-needle aspiration, birth: Fine-needle biopsy: The changing trend in endoscopic ultrasound-guided tissue acquisition. Dig Endosc 2019; 31: 197-202

[21] Naveed M, Siddiqui AA, Kowalski TE et al. A multicenter comparative trial of a novel EUS-guided core biopsy needle (SharkCoreTM) with the 22-gauge needle in patients with solid pancreatic mass lesions. Endosc Ultrasound 2018; 7: 34-40

[22] Song Z, Trujillo CN, Song $\mathrm{H}$ et al. Endoscopic ultrasound-guided tissue acquisition using fork-tip needle improves histological yield, reduces needle passes, without on-site cytopathological evaluation. J Pancreat Cancer 2018; 4: 75-80

[23] Bang JY, Hebert-Magee S, Navaneethan U et al. Randomized trial comparing the Franseen and Fork-tip needles for EUS-guided fineneedle biopsy sampling of solid pancreatic mass lesions. Gastrointest Endosc 2018; 87: 1432-1438 